\title{
Driving experience in a natural context of racing video game players does not affect gaze strategy towards tangent point during simulated curve negotiation, but the curvature angle does
}

\author{
GABRIEL P. PASCHOALINO1 | GABRIEL K. KUGA² | GISELE C. GOTARDI',2 | RAFAEL O. SIMÃO ${ }^{1,2}$ | FABIO A. \\ BARBIERI ${ }^{1,2,3}$ | MARTINA NAVARRO ${ }^{4}$ | PAULA F. POLASTRI ${ }^{1,2}$ | SÉRGIO T. RODRIGUES ${ }^{1,2}$ | \\ ${ }^{1}$ Laboratory of Information, Vision and Action, Department of Physical Education, Faculty of Sciences, São Paulo State University, Bauru, São Paulo, Brazil. \\ 2 Human Movement Science Graduate Program, Department of Physical Education, Faculty of Sciences, São Paulo State University, Bauru, São Paulo, Brazil. \\ 3 Human Movement Research Laboratory (MOVI-LAB), Department of Physical Education, Faculty of Sciences, São Paulo State University, Bauru, São Paulo, Brazil. \\ ${ }^{4}$ Department of Sport and Exercise Science, Faculty of Science, University of Portsmouth, Portsmouth, England.
}

Correspondence to: Sérgio T. Rodrigues

email: sergio.tosi@unesp.br

https://doi.org/10.20338/bjmb.v13i4.137

\section{HIGHLIGHTS \\ - Gaze strategy towards tangent point is not affected by natural context experience. \\ - Curvature angles modified fixation patterns. \\ - Visual demands increased during sharper curves in simulated curve negotiation. \\ ABBREVIATIONS \\ AOI Areas of Interest \\ DR Drivers \\ Gamers who regularly play racing video games \\ ND Non-Drivers \\ TP tangent point}

\section{PUBLICATION DATA}

Received 07062019

Accepted 16082019

Published 01102019

\begin{abstract}
BACKGROUND: During curve negotiation, drivers tend to look at the tangent point of the curve (TP) to control steering. There is a lack of evidence on how experiences of natural and simulated driving are related.

AIM: This study aimed to investigate the effects of experience in natural driving on gaze behavior of racing video game players. Additionally, we aimed to identify possible effects of curvature angle on fixation patterns.

METHOD: Twenty video game players composed two groups: driver-gamers and non-driver-gamers. The gaze of participants was recorded during performance of three laps racing on a video game circuit, which included eleven curves. Areas of interest were defined relative to TP: 3 degrees of visual angle, 10 degrees of visual angle, and others. Dependent variables were: Number of Fixations and Total Duration of Fixations.

RESULTS: Results showed that driving experience in a natural context did not change TP visual strategy during curve negotiation in a racing video game environment. All participants spent approximately half of their number of fixations and total fixation time within 10 degrees around the TP. Curvature angles modified fixation patterns, causing a higher number of fixations and total fixation time during sharper curves.

CONCLUSION: The results indicate that natural driving experience does not change gamers' gaze behavior during simulated curve negotiation while visual information acquisition from the TP was modulated by curvature angle.
\end{abstract}

\section{INTRODUCTION}

The role of vision during driving has been studied for many years ${ }^{1,2,3}$. Advances in technology have enabled the recording of eye movements during a variety of tasks in natural and simulated environments (e.g., Gotardi et al. $(2019)^{4}$, Ciceri et al. $(2014)^{5}$, Lehtonen et al. (2013)3 ${ }^{3}$ Konstantopoulos, Chapman, \& Crundall (2010)6). Highly complex visuomotor tasks, such as driving a vehicle, require sophisticated coordination between superior and inferior limbs, as well as gaze orientation with respect to the lane?.

During curve negotiations, drivers direct their gaze fixations towards the tangent point (TP), defined as the most prominent point on the inner side of the bend where the driver's line of gaze is tangential to the road edge 2 . Land and Lee $(1994)^{2}$ investigated steering control on a road with steep curves, recording the steering wheel angle and the 
drivers' gaze direction, and found that before the car entered the bend and during the bend, drivers spent a lot of time fixating on the TP; a clear correlation between gaze and steering wheel angle occurred, with eye movements preceding the rotation of the steering wheel by approximately $0.8 \mathrm{~s}$, suggesting that gaze angle directed at the TP prior to entering and during bends can provide informational knowledge needed to control the steering wheel ${ }^{2,8}$. This finding has been confirmed by more recent studies, although complementary and alternative explanations are still under debate ${ }^{9,10,11}$. Interestingly, Land and Lee's findings were associated with the use of winding country roads, one-way and single lane (without the distraction of other traffic), where the curvature changed continuously and unpredictably ${ }^{8}$. This makes lane edge feedback on its own a less efficient basis for steering and estimating values of future curvature; the authors pointed out that earlier studies had relatively shallow curvatures and found only a weak relationship between gaze direction and steering control (e.g., Zwahlen (1993)12). The authors also explained that during gentle bends of constant curvature, simple lane position maintenance (based on visual feedback from local splay angles) enables rapid steering to adapt to road curvature, without explicit estimates of bend curvature, allowing motor adjustments as necessary. Under these circumstances, although feed forward (anticipatory) estimates of future curvature from either road features or flow lines can supplement this process, they may not be necessary at moderate speeds ${ }^{8}$.

Few studies have focused on the effects of experience on curve negotiation during driving. Lehtonen et al. (2013) ${ }^{3}$ classified two types of fixations during curves; guiding fixations to collect information relative to the ongoing task (e.g., guiding the vehicle through the curve), and look-ahead fixations to support prediction of how the parameters associated with the task will behave in the near future (e.g., an upcoming corner or information from the TP). Experienced and novice drivers were asked to drive an instrumented vehicle on a rural road while their eye movements were recorded. The experienced drivers spent less time performing fixations towards the oncoming road and more time on the look-ahead fixations over the curves. The authors suggested that with driving experience, peripheral vision can be increasingly used in the visual guidance of steering. This could allow experienced drivers to spend more time of their visual attention on look-ahead fixations over curves, facilitating trajectory planning and detection of the TP3. It has been shown that experienced drivers perform a greater number of shorter fixations with a wider horizontal spread across the visual scene when compared to novice drivers ${ }^{13,6}$, indicating that driving experience plays a critical role in driving. However, there is a dearth of evidence on whether driving experience influences TP gaze strategy during curve negotiation in a simulated environment (e.g., driving simulators, racing video games).

Video games represent a relevant environment to investigate perception-action functioning. It is known that video game practice improves cognitive and perceptual-motor skills ${ }^{14}$ and that video game players react faster than non-video game players in a variety of tasks ${ }^{15}$. In addition, it is well established in the motor behavior literature that practice and experience induce improvement in perceptual-motor performance and that context similarities facilitate learning transfer (e.g., Magill $(2013)^{16}$, Schmidt $\left.(2008)^{17}\right)$. However, how these general principles apply to natural and simulated driving and gaze behavior is a matter of debate. The aim of this study was to investigate the effects of driving experience in a natural context on gaze behavior in experienced video game players in a simulated driving task. Firstly, we hypothesized that experienced drivers who regularly play racing video games (gamers) would perform a higher number of fixations and spend more time 
fixating towards the TP during curve negotiation compared to the non-driver gamers; we expected that this effect of driving experience on gaze strategy towards the TP during curve negotiation would also be present in the simulated video game environment. Secondly, we hypothesized that steeper curvatures would increase the need for information acquisition in the tangent area, causing a higher number and total duration of fixations during curve negotiation in a simulated context.

\section{METHODS}

\section{Participants}

Twenty young adults voluntarily participated in this study, being equally divided into two groups: Drivers (DR; $24.5 \pm 2.36$ years, $84.6 \pm 18.9 \mathrm{~kg}, 176.9 \pm 3.7 \mathrm{~cm}$ ) and NonDrivers (ND; $19.5 \pm 3.74$ years, $65.9 \pm 10.7 \mathrm{~kg}, 168.4 \pm 6.9 \mathrm{~cm}$ ). All participants presented a similar experience profile, declaring regular practice in racing video games with a minimum frequency of three times per week and one hour per session. Participants were informed about the objectives and procedures of the experiment, and reported no muscular, neurological, postural, or locomotor diseases. All participants had normal visual acuity or corrected to normal by glasses or contact lenses. Participants in the DR group held a Brazilian driver's license with minimum driving experience of four years in natural, "real world" situations. Ethical approval for the study was obtained from the Local Committee and participants signed an informed consent before starting the study.

\section{Apparatus and procedures}

Participants were seated in a chair in front of a regular table (width $=130 \mathrm{~cm} \mathrm{x}$ depth $=59.5 \mathrm{~cm} \times$ height $=75 \mathrm{~cm}$ ) with the following pieces of equipment attached and correctly positioned: gear-shifter, steering wheel, and accelerator, brake, and clutch pedals (Logitech G27), as shown in Figure 1C. The chair back rest was positioned $2 \mathrm{~m}$ from the projection screen (204 cm x $154 \mathrm{~cm}$, Projetelas Infinity) where the video game image was projected (projector BenQ MX720). The racing video game Gran Tourism (version 4), running on Playstation 2 (Sony), was set with the following configurations: Arcade Mode, Time Trial, Original Circuits, Autumn Ring Mini, and Ford Ka 01. Participants' eye movements were recorded by a head-mounted eye tracker (model H6, ASL) at a sampling rate of $60 \mathrm{~Hz}$.

Participants were free to adjust the distance of the steering wheel and pedals in order to allow a comfortable driving posture. All participants were allowed five-minutes of driving to familiarize themselves with the equipment and software. The eye tracker was adjusted to each participant's head and a nine-point calibration was conducted to accurately measure the participant's point of gaze throughout the trial. Crossed lines in Figure 1B represent point of gaze during curve negotiation. A regular television (Sony Triniton) was used to reproduce the audio generated by the racing video game. Participants were instructed to complete three laps in the game circuit (Autumn Ring Mini) as fast as possible. This circuit contained eleven curves with distinct curvature angles, as shown in Figure $1 \mathrm{~A}$. 


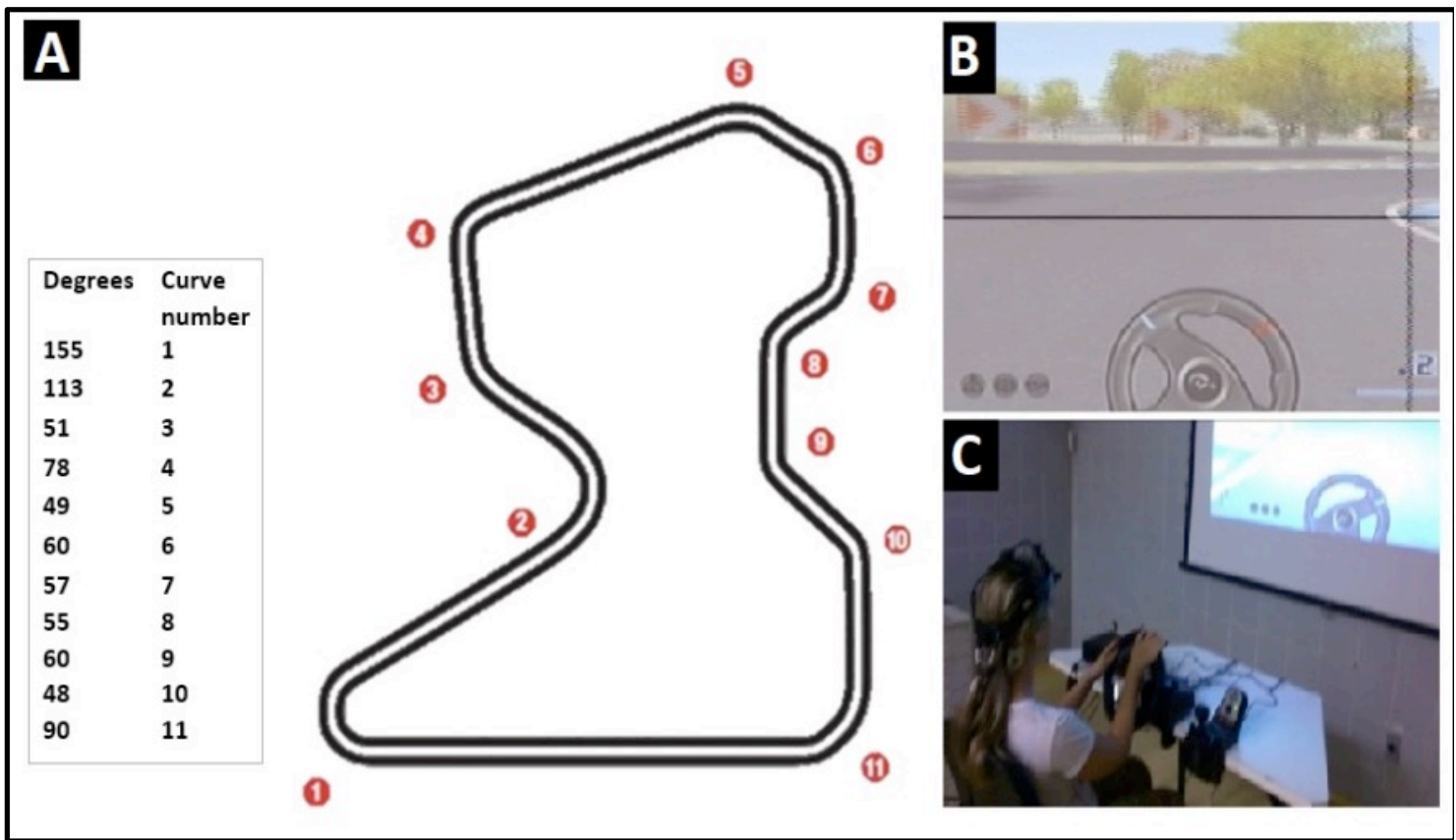

Figure 1. (A) The Autumn Ring Mini circuit, on which participants performed the task (http://granturismo.wikia.com/wiki/File:Autumn_Ring_Mini_Forward.png); (B) Example frame of video scene during a curve negotiation within the racing video game task, showing a superimposed black cursor (horizontal and vertical lines) which represents the participant's line of gaze; and (C) Photograph of the experimental setup.

\section{Data Analysis}

Gaze recordings were transferred to a PC (ASUS) running ASL Results Plus software (version 1.8.2.18, ASL). The videos were analyzed through Areas of Interest (AOI) analyses. AOls were two-dimensional (2-D) rectangular regions defined in the viewing layout (e.g., eye tracker scene video) in which the gaze fixation patterns (number and duration of fixations) were identified in relevant parts of the visual scene. Three AOls were defined: i) 3 degrees from the TP; ii) 10 degrees from the TP, and iii) Others - all portions of the visual scene that were not considered an AOI. Although foveal vision is usually defined within two or three degrees of visual angle, due to anatomical distribution of cones over the retina, an alternative area of 10 degrees was used based on evidence of the utility of parafoveal processing (e.g., Fiorentini (1989) ${ }^{18}$, Traschütz, Zinke, \& Wegener $\left.(2012)^{19}\right)$. The center of the AOls of 3 and 10 degrees was located on the TP, which was defined as the point inside the curve on the lane edge where the line of sight is tangential to the lane edge, and corresponds to the point in the driver's visual field where the visual orientation of the projection of the edge line is reversed ${ }^{11}$. The same angular length of each area was utilized in both horizontal and vertical axes. Rectangular regions of the scene defined as AOls were calculated a priori based on the size of the projected image and drivers' distance from it. Each $\mathrm{AOI}$ was manually positioned frame-by-frame relative to the TP in order to maintain correct locations while the visual path was constantly moving throughout the curves.

Gaze analysis was based only on the video frames of each curve, excluding rectilinear trajectories. Curves were taken to be between the first look at the TP, and the disappearance of the TP at the exit from the curve? ${ }^{2}$. 
The dependent variables, resulting from the mean value of three laps in the circuit with respect to each AOI, were defined as follows: Number of Fixations (units) - total number of fixations identified during each curve; and Total Duration of Fixations (s) - sum of duration of all fixations identified during each curve. The criteria adopted for gaze fixation was when two times the point of gaze standard deviation (95\% confidence interval) was less than one degree of visual angle (horizontal and vertical) over $100 \mathrm{~ms}$. Zero values represented trials in which no fixations occurred in a given $\mathrm{AOl}$ for both number and duration of fixation variables. An additional analysis quantified this absence of fixations, attributing values of zero (no fixations) and one (with fixations) to each design cell. Each dependent variable was submitted to a Group (Drivers, Non-Drivers) by AOI ( 3 degrees, 10 degrees, others) by Curve (Curves 1 to 11) analysis of variance with repeated measures in the last two factors. Tests of contrast were conducted as post hoc tests specifically to evaluate trends in Curvature Angle effect. Post-hoc Tukey HSD tests, Greenhouse-Geisser degrees of freedom adjustments, and Bonferroni multiple comparison probability adjustments were conducted as necessary ${ }^{20}$. The alpha value was set at 0.05 for all analyses. Effect sizes were calculated using Partial Eta Squared with 0.02 or less, approximately 0.13 , and 0.26 or more, representing small, medium, and large effect sizes, respectively ${ }^{21}$. Matlab software (The Matworks Inc., 2010- version 7.10.0.499) was used for necessary calculations and SPSS (SPSS Inc., 2008 - version 17.0.1) for statistical analysis.

\section{RESULTS}

Total fixation duration was significantly affected by AOI, $F(2,36)=18.32, p<.001$, $\eta^{2}=.504$, and by Curve, $F(1.2,21.0)=5.34, p=.027, \eta^{2}=.229$. Post-hoc tests showed that total fixation duration was significantly smaller in $\mathrm{AOI}$ of 3 degrees than in $\mathrm{AOI}$ of 10 degrees $(p<.001)$ and in AOI others $(p<.001)$, as shown in Figure 2A. The Post hoc test of the curvature angle effect indicated a significant linear trend, $F(1,18)=56.15, p<.001$, $\eta^{2}=.757$, demonstrating that total fixation duration increased as curvature angle increased, as shown in Figure $2 \mathrm{~B}$. The curvature angle was capable of explaining $51 \%$ of the variation in total fixation duration values. 

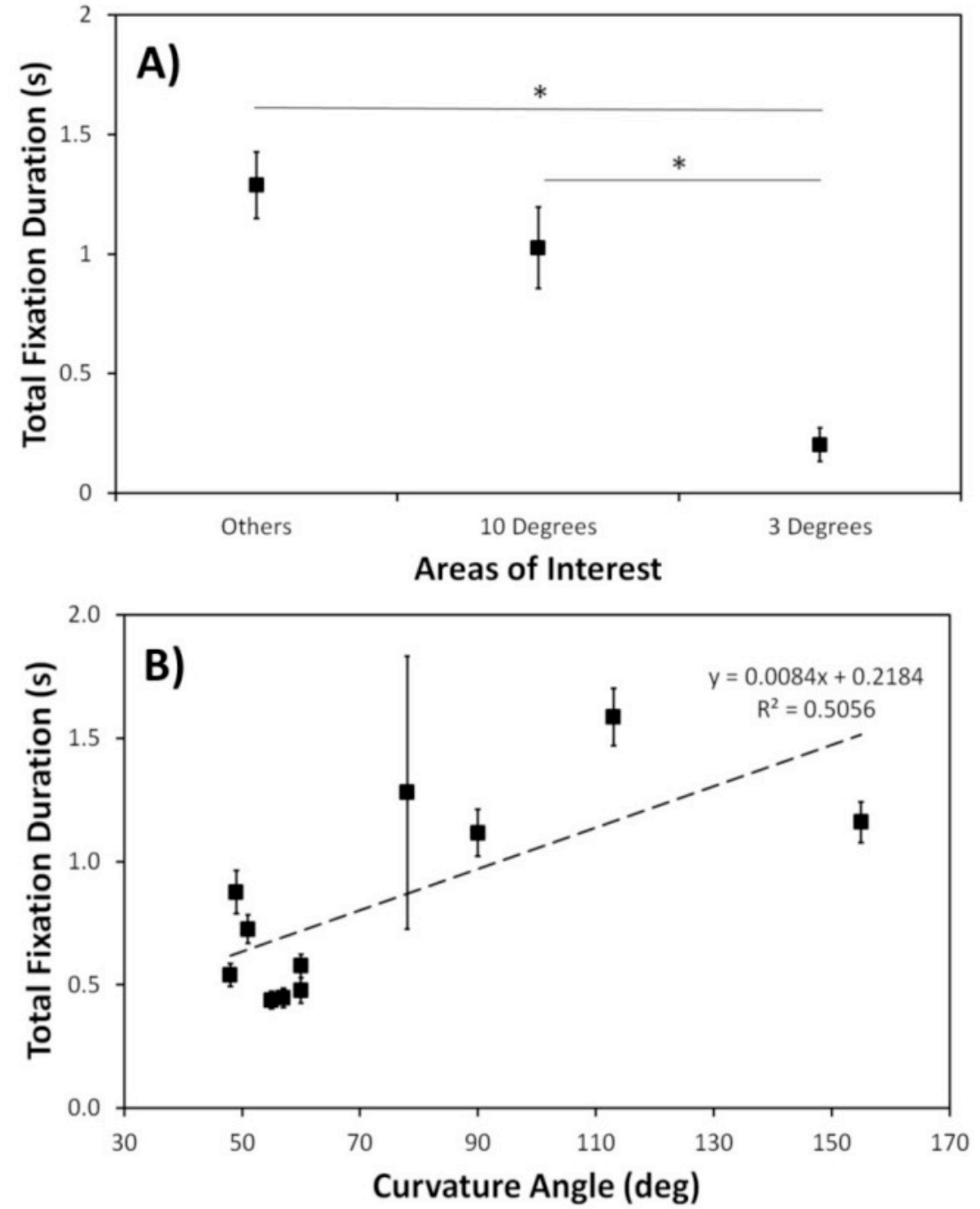

Figure 2. Total Fixation Duration (s) for (A) areas of interest of 3 degrees, 10 degrees, and others [ ${ }^{*}$ Significant difference at .05 level] and (B) as a function of curvature angles (degrees) [Regression line shown as dashed line].

Number of fixations was significantly affected by $\mathrm{AOI}, F(2,36)=29.53, p<.001$, $\eta^{2}=.621$, by Curve, $F(10,180)=22.19, p<.001, \eta^{2}=.552$, and $\mathrm{AOI}$ by Curve interaction, $F(5.5,11.5)=18.32, p=.002, \eta^{2}=.178$. Post-hoc tests showed that number of fixations was significantly smaller in $\mathrm{AOI}$ of 3 degrees $(M=.70, S E=.20)$ than in $\mathrm{AOI}$ of 10 degrees $(M=3.98, S E=.48)$ and in $A O I$ others $(M=3.96, S E=.32), p<.001$. Additionally, posthoc tests revealed significant effects of curve on the number of fixations in AOls of 3 degrees $(p=.009), 10$ degrees ( $p<.001)$, and others ( $p<.001)$. Post hoc tests of the curvature angle effect conducted separately for each $\mathrm{AOI}$ indicated significant linear trends for AOI of 3 degrees, $F(1,18)=12.34, p=.002, \eta^{2}=.407$ (Figure $3 C$ ), AOl of 10 degrees, $F(1,18)=19.84, p<.001, \eta^{2}=.524$ (Figure 3B), and AOI others, $F(1,18)=44.37$, $p<.001, \eta^{2}=.711$ (Figure 3A), demonstrating that number of fixations increased as curvature angle increased in each $\mathrm{AOI}$, with the curvature angle being capable of explaining, respectively, 63,42 , and $54 \%$ of the variation in the number of fixations values. 

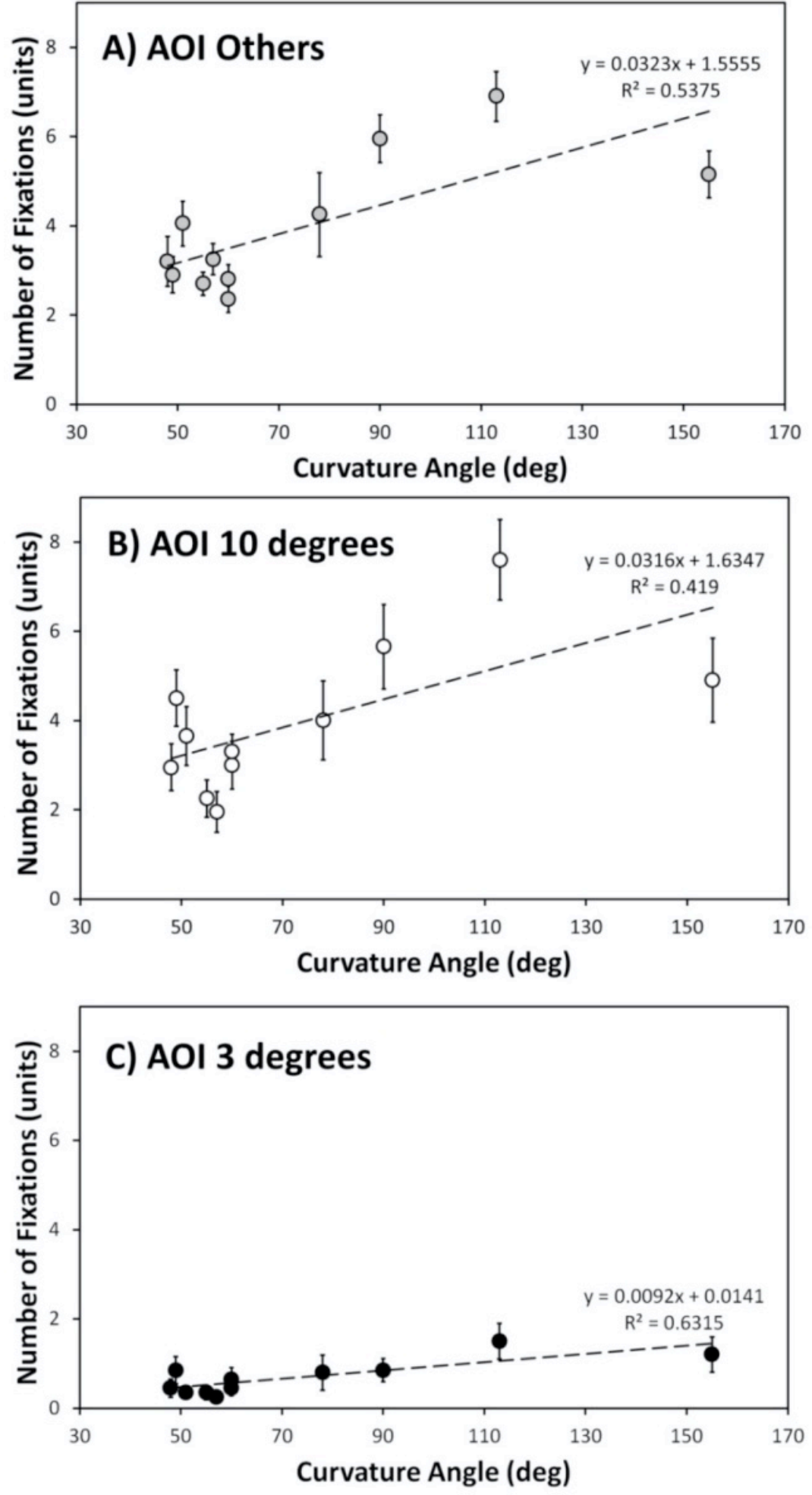

Figure 3. Number of Fixations (units) as a function of curvature angles (degrees) for areas of interest (A) others, (B) 10 degrees (B), and (C) 3 degrees [Regression lines shown as dashed lines]. 
The analysis regarding the absence of fixations (through the use of zero and one values) revealed a significant effect of $\mathrm{AOI}, F(2,36)=56.61, p<.001, \eta^{2}=.759$, showing that absence of fixations was significantly higher in the AOI of 3 degrees $(M=.491$, SE $=.066)$ than in the $\mathrm{AOI}$ of 10 degrees $(M=.927, \mathrm{SE}=.022)$ and others $(M=.995$, SE $=.005$ ), $p<.001$; the AOI of 10 degrees also demonstrated a significantly higher absence of fixations than $\mathrm{AOI}$ others, $p=.026$. All unreported effects or interactions on dependent variables did not reach significance level.

\section{DISCUSSION}

The purpose of this study was to analyze the effects of driving experience in a natural context on the utilization of information from the TP during simulated curve negotiation in a video game situation. Overall, the results do not corroborate the expectation that more experience of "real world" driving would increase the number and total duration of fixations during curve negotiation in the video game context (first hypothesis); both groups performed similarly in the task. Differently, sharper curves demanded more attention from both groups, as revealed by linear trend analysis of gaze variables, confirming our second hypothesis. First, we focus on the overview of the TP strategy in the present task, after which we discuss the effects of experience and curvature.

Land and Lee (1994)2 showed that although off-road glances are possible and tend to vary with road curvature and with the stage of the bend that the vehicle has reached, when entering a new bend, drivers fixated on the TP around $80 \%$ of the time, which characterizes the need for attention to presumably obtain a correct initial estimate of the curvature of the bend. In addition, Land and Lee, using contour plots of the density of fixations (with higher densities closer to the TP), showed that $65 \%$ of the fixations were concentrated on the contours and the rest was widely spread on the visual scene. The present study found that participants' total fixation duration represented $48.8 \%$ of their fixation time around the TP (combining $7.9 \%$ within 3 degrees and $40.9 \%$ within 10 degrees) while $51.2 \%$ were on other areas. Similarly, $54.2 \%$ of the number of fixations occurred around the TP (combining $8.1 \%$ within 3 degrees and $46.1 \%$ within 10 degrees) while $45.8 \%$ were on other areas. These findings suggest that participants did not exclusively utilize the fovea to obtain information from the bend curvature to perform the task. As focal vision covers only a small fraction of visual field, its utilization may not always be sufficient to provide useful information to the driver to perform the maneuver. This is in line with previous studies showing that parafoveal ability to discriminate useful information related to specific tasks can triplicate with training 22 . Overall, the present study seems to confirm that participants used the TP strategy during simulated curve negotiation, spending around half of their number and total duration of fixations on the tangent area. Although these results are relatively low compared to the findings of Land and Lee (54 vs. $65 \%$ and 49 vs. $80 \%$, respectively), the present study took place in a virtual (video game) environment and most curves were moderate in curvature ( 7 out of 11 curves were below 60 degrees), aspects expected to decrease attentional effort and gaze fixation features towards the tangent area.

Our initial expectation was that experienced drivers would perform a greater number and total duration of fixations towards the TP than non-drivers due to their practice in the natural context. However, there were no differences between groups. One possible 
explanation for understanding the similarity between driver-gamers and non-driver-gamers is that both groups had experience in the racing video game environment. In the current study, participants' gaze behavior seemed guided by the task specificity rather than driving experience in a natural context. As drivers become more experienced in driving tasks, changes in their visual search strategy involve making longer fixations on the most relevant parts of the scene, especially in situations with potential hazards, when quick decision making is necessary ${ }^{23}$. In the same vein, participants with experience in driving vehicles in a simulated context (video game) may have developed particular visual exploration strategies relative to this task. The results indicate that specificity between video game experience and experimental task demands was predominant to TP utilization during bend maneuver; differently, experience in a natural driving context did not affect the pattern of TP fixations in the simulated context.

Our second hypothesis was confirmed. Curvature angle altered gaze patterns, suggesting change in attention requirements from both groups. Linear trend analysis of gaze fixation variables revealed that sharper curves were significantly associated with a higher number of fixations and total fixation durations. This agrees with previous results showing that visual demand and the inverse of curve radius were reciprocal at constant speed, with sharper curves increasing the need for visual information ${ }^{24}$. In addition, motor demand increases when driving in a sharper curve, as faster deviations from lane can occur, which indicates that TP utilization is linked to maneuver planning.

\section{CONCLUSION}

Natural context driving experience did not change the TP visual strategy during curve negotiation in a racing video game environment. All participants spent approximately half of their number of fixations and total fixation time within 10 degrees around the TP. Curvature angles modified fixation patterns, causing a higher number of fixations and total fixation time during sharper curves. The findings of the present study have relevant implications related to how natural and virtual visual stimuli contexts are mutually transferable in terms of driving perceptual and motor learning, as well as regarding technological applications for the automotive industry and traffic safety. Theoretically, knowledge revealed by this study on the particular, well-known gaze strategy (TP based curve negotiation theory) confirms reports on visual attention in the literature and adds novelty regarding the role of curvature angle in driving. Practically, the present study explored principles related to the use of simulation classes in driving schools (as recently required in the driving licensing process in Brazilian Traffic Law), showing that the visual control of curve negotiation during natural driving is similarly maintained in the simulated context.

\section{REFERENCES}

1. Donges, E. A Two-Level Model of Driver Steering Behavior. Human Factors: The Journal of the Human Factors and Ergonomics Society. 1978; 20(6), 691-707.

2. Land M. F., \& Lee, D. N. Where we look when we steer. Nature. 1994; 369(6483):742-44. 
3. Lehtonen, E., Lappi, O., Kotkanen, H., \&Summala, H. Look-ahead fixations in curve driving. Ergonomics. 2013; 56(1), 34-44.

4. Gotardi, G. C., Polastri, P. F., Schor, P., Oudejans, R. R. D., van der Kamp, J., Savelsbergh, G. J. P., Navarro, M., Rodrigues, S. T. Adverse effects of anxiety on attentional control differ as a function of experience: A simulated driving study. Applied Ergonomics. 2019; 74, 41-47.

5. Ciceri MR, et al. Does driving experience in video games count? Hazard anticipation and visual exploration of male gamers as function of driving experience. Transportation Research Part F. 2014; 22:76-85.

6. Konstantopoulos, P., Chapman, P., \&Crundall, D. Driver's visual attention as a function of driving experience and visibility. Using a driving simulator to explore drivers' eye movements in day, night and rain driving. Accident Analysis \& Prevention, 2010; 42(3), 827-834.

7. Land, M. F. Eye movements and control of actions in everyday life. Progress in Retinal and Eye Research. 2006; 25(3):296-324.

8. Land, M. F., \&Tatler, B. W. Looking and acting: Vision and eye movements in natural behaviour. Oxford, UK: Oxford University Press, 2009.

9. Authié $\mathrm{CN}$, et al. Optokineticnystagmus is elicited by curvilinear optic flow during high speed curve driving. Vision Research, 2011; 51(16):1791-8000.

10. Mars, F., \& Navarro, J. Where We Look When We Drive with or without Active Steering Wheel Control. PLoS ONE, 2012; 7(8), e43858.

11. Lappi, $O$. Future path and tangent point models in the visual control of locomotion in curve driving. Journal of Vision, 2014; 14(12):21, 1-22.

12. Zwahlen, H.T. Eye scanning rules for drivers: how do they compare with actual observed eye-scanning behavior? Transportation Research Record, 1993; 1403, 14-22.

13. Crundall, D. E., \& Underwood, G. Effects of experience and processing demands on visual information acquisition in drivers. Ergonomics, 1998; 41(4), 448-458.

14. Powers, K. L., Brooks, P. J., Aldrich, N. J., Palladino, M. A., \& Alfieri, L. Effects of videogame play on information processing: A meta-analytic investigation. Psychonomic Bulletin \& Review, 2013; 20(6), 1055-1079.

15. Mack, D. J., \&llg, U. J. The effects of video game play on the characteristics of saccadic eye movements. Vision Research, 2014; 102, 26-32.

16. Magill, R. A., \& Anderson, M. Motor Learning and Control: Concepts and Applications: Tenth Edition. McGraw-Hill Higher Education, 2013.

17. Schmidt, R. A., \&Wrisberg, C. A. Motor Learning and Performance: a situation-based learning approach. Fourth Edition. Human Kinetics, 2008.

18. Fiorentini, A. Differences between fovea and parafovea in visual search processes. Vision Res. 1989; Vol. 29, No. 9, 1153-1164.

19. Traschütz, A., Zinke, W., \& Wegener, D. Speed change detection in foveal and peripheral vision. Vision Research, 2012; 72, 1-13. 
Brazilian Journal of Motor Behavior

20. Maxwell, S. E., \& Delaney, H. D. Designing experiments and analyzing data: A model comparison perspective. Belmont, CA, US: Wadsworth/Thomson Learning, 1990.

21. Cohen, J. Statistical Power Analysis for the Behavioral Sciences. 2nd Ed. Lawrence Ebarum Associates, 1988.

22. Beard, B. L., Levi, D. M., \& Reich, L. N. Perceptual learning in parafoveal vision. Vision Research, 35(12), 1995; 1679-1690.

23. Chapman $P$, et al. Visual search patterns in trained and untrained novice drivers. Transportation Research Part F, 2002; 5(2):157-67.

24. Wooldridge, M., Fitzpatrick, K., Koppa, R., \& Bauer, K. Effects of Horizontal Curvature on Driver Visual Demand. Transportation Research Record: Journal of the Transportation Research Board, 2000; 1737, 71-77.

Citation: Paschoalino GP, Kuga GK, Gotardi GC, Simão RO, Barbieri FA, Navarro M, Polastri PF, Rodrigues ST.

Racing video game players' driving experience in natural context does not affect gaze strategy towards tangent point during simulated curve negotiation, but the curvature angle does. BJMB.2019:13(4): 113-123.

Editor: Dr José Angelo Barela - São Paulo State University (UNESP), Rio Claro, SP, Brazil; Dr Natalia Madalena Rinaldi - Federal University of Espírito Santo (UFES), Vitória, ES, Brazil.

Copyright: @ 2019 Paschoalino, Kuga, Gotardi, Simão, Barbieri, Navarro, Polastri and Rodrigues and BJMB. This is an open-access article distributed under the terms of the Creative Commons Attribution-NonCommercial-NoDerivatives 4.0 International License which permits unrestricted use, distribution, and reproduction in any medium, provided the original author and source are credited.

Funding:This work was supported by CNPq (Conselho Nacional de Desenvolvimento Científico e Tecnológico), Brazil (grant number 458916/2014-5).

Competing interests: The authors have declared that no competing interests exist.

DOI: https://doi.org/10.20338/bjmb.v13i4.137 